\title{
Exosome-based biomimetic nanoparticles targeted to inflamed joints for enhanced treatment of rheumatoid arthritis
}

\author{
Feili Yan ${ }^{1 \dagger}$, Zhirong Zhong ${ }^{1 \dagger}$, Yao Wang ${ }^{1 \dagger}$, Yue Feng ${ }^{2,3}$, Zhiqiang Mei ${ }^{4}$, Hui Li ${ }^{1}$, Xiang Chen ${ }^{1}$, Liang Cai ${ }^{2,3^{*}}$ \\ and Chunhong $\mathrm{Li}^{1,5^{*}}$
}

\begin{abstract}
Background: Glucocorticoids (GCs) show powerful treatment effect on rheumatoid arthritis (RA). However, the clinical application is limited by their nonspecific distribution after systemic administration, serious adverse reactions during long-term administration. To achieve better treatment, reduce side effect, we here established a biomimetic exosome (Exo) encapsulating dexamethasone sodium phosphate (Dex) nanoparticle (Exo/Dex), whose surface was modified with folic acid (FA)-polyethylene glycol (PEG)-cholesterol (Chol) compound to attain FPC-Exo/Dex active targeting drug delivery system.
\end{abstract}

Results: The size of FPC-Exo/Dex was $128.43 \pm 16.27 \mathrm{~nm}$, with a polydispersity index (PDI) of $0.36 \pm 0.05$, and the Zeta potential was $-22.73 \pm 0.91 \mathrm{mV}$. The encapsulation efficiency (EE) of the preparation was $10.26 \pm 0.73 \%$, with drug loading efficiency (DLE) of $18.81 \pm 2.05 \%$. In vitro study showed this system displayed enhanced endocytosis and excellent anti-inflammation effect against RAW264.7 cells by suppressing pro-inflammatory cytokines and increasing anti-inflammatory cytokine. Further biodistribution study showed the fluorescence intensity of FPC-Exo/Dex was stronger than other Dex formulations in joints, suggesting its enhanced accumulation to inflammation sites. In vivo biodistribution experiment displayed FPC-Exo/Dex could preserve the bone and cartilage of CIA mice better and significantly reduce inflamed joints. Next in vivo safety evaluation demonstrated this biomimetic drug delivery system had no obvious hepatotoxicity and exhibited desirable biocompatibility.

Conclusion: The present study provides a promising strategy for using exosome as nanocarrier to enhance the therapeutic effect of GCs against RA.

Keywords: Dexamethasone sodium phosphate, Biomimetic, Exosomes, Folic acid, Rheumatoid arthritis

*Correspondence: cllc131420@sina.com; lispringhong@126.com

${ }^{\dagger}$ Feili Yan, Zhirong Zhong and Yao Wang contributed equally to this work.

${ }^{1}$ Department of Pharmaceutical Sciences, School of Pharmacy,

Southwest Medical University, 3-319 Zhongshan Road, 646000 Luzhou,

Sichuan, People's Republic of China

${ }^{2}$ Department of Nuclear Medicine, The Affiliated Hospital of Southwest

Medical University, 3-319 Zhongshan Road, 646000 Luzhou, Sichuan,

People's Republic of China

Full list of author information is available at the end of the article

\section{Introduction}

Rheumatoid arthritis (RA) is a chronic autoimmune disease whose pathophysiology is unclear [1]. As it progresses, articular cartilage and bone are destroyed, which can cause disability eventually $[2,3]$. This progression may be driven by pro-inflammatory cytokines such as tumor necrosis factor (TNF)- $\alpha$ and the interleukins (IL)-1 $\beta$ and IL-6, as well as the inflammatory mediators inducible nitric oxide synthase and epoxidase [4, 5]. Glucocorticoids (GCs), the most widely

(c) The Author(s) 2020. This article is licensed under a Creative Commons Attribution 4.0 International License, which permits use, sharing, adaptation, distribution and reproduction in any medium or format, as long as you give appropriate credit to the original author(s) and the source, provide a link to the Creative Commons licence, and indicate if changes were made. The images or other third party material in this article are included in the article's Creative Commons licence, unless indicated otherwise in a credit line to the material. If material is not included in the article's Creative Commons licence and your intended use is not permitted by statutory regulation or exceeds the permitted use, you will need to obtain permission directly from the copyright holder. To view a copy of this licence, visit http://creativeco mmons.org/licenses/by/4.0/. The Creative Commons Public Domain Dedication waiver (http://creativecommons.org/publicdomain/ zero/1.0/) applies to the data made available in this article, unless otherwise stated in a credit line to the data. 
used first-line drugs against RA, can control inflammation and relieve pain quickly by inhibiting the secretion of pro-inflammatory cytokines and up-regulating anti-inflammatory protein IL-10 [6,7], which further inhibits the secretion of pro-inflammatory cytokines. However, long-term use of systemic, high-dose glucocorticoids is associated with serious adverse effects, such as decreased immunity, hyperglycemia and osteoporosis [8].

Delivering anti-RA drugs within nanoparticles may help target the drugs to inflamed tissues, thereby improving therapeutic efficacy and reducing adverse effects $[8$, 9]. Nanoparticles can accumulate at inflammatory sites through a process known as "extravasation through leaky vasculature and subsequent inflammatory cell-mediated sequestration" (ELVIS), which is analogous to the "enhanced permeability and retention" (EPR) effect that can lead nanoparticles to accumulate in solid tumors.

Indeed, targeted delivery of glucocorticoids against RA has already been achieved using liposomes, physically encapsulated micelles and polymer nanoparticles [2, 10-12]. However, liposomes are poor at evading host immune system clearance, and they show instability and short circulation time [13, 14]. Physically encapsulated micelles are unstable in circulation because they are diluted and they can interact with proteins and other components of plasma [2,3]. Polymer nanoparticles may be more stable than liposomes and physically encapsulated micelles, but their long-term biocompatibility and safety pose challenges $[2,5]$. Consequently, developing more reliable and powerful nanocarriers is highly desired.

Exosomes have emerged as an alternative to those exogenous nanoparticles $[15,16]$. These membrane-enclosed vesicles with sizes of 40-150 nm are naturally secreted by various cell types, which endow them with fascinating natural properties such as low cytotoxicity, non-immunogenicity, desirable biocompatibility, specific targeting capacity and prolonged systemic circulating ability [15, 17-19]. These superior properties making them ideal drug delivery nanocarriers. Moreover, exosome-based nanoparticles drug delivery has been successfully applied in loading various drugs for different diseases treatment, such as cancer, Parkinson, renal and brain inflammation, but has been rarely used in RA so far [19-22]. However, the exosome-based drug delivery existed flaw in selective accumulation to target sites in vivo [21-23].

Study on the pathological microenvironment of RA suggested that inflamed areas contain abundant activated macrophages that express folic acid receptors (FRs) on their surface, particularly $\operatorname{FR} \beta[24,25]$. Therefore, we reasoned that it may be effective to construct exosome-based nanoparticles coating with folic acid (FA) for enhancing accumulating ability by active targeting effect to FR $\beta$ in vivo. Therefore, we used exosomes to encapsulate dexamethasone sodium phosphate (Dex), one of the most frequently used GCs to treat RA in clinical, to obtain Exo/ Dex nanoparticle firstly. Then, we modified it with FApolyethylene glycol (PEG)-cholesterol (Chol) compound (FPC) to prepare FPC-Exo/Dex active targeting drug delivery. Meanwhile liposome drug delivery system (Lip/Dex) was fabricated for comparative study to Exo/Dex in this research. Properties of these nanoparticles were characterized in vitro. Then the internalization and anti-inflammatory effects to lipopolysaccharide-activated RAW264.7 cells were examined. Furthermore, their biodistribution, therapeutic efficacy against RA and in vivo safety were studied in mice with collagen-induced arthritis (CIA).

\section{Material and methods \\ Materials}

Dexamethasone sodium phosphate was supplied by Solarbio Science \& Technology (Beijing, China). Methanol and acetonitrile (HPLC grade) were purchased from Kelong Chemical Reagent Factory (Chengdu, China). Rabbit monoclonal antibodies against CD63 or CD9 as well as horseradish peroxidase-conjugated goat antirabbit IgG were purchased from Abcam (UK). SDSPAGE kits and polyvinylidene fluoride membranes were obtained from Sigma (USA). Complete and incomplete Freund's adjuvant and bovine type II collagen were acquired from Chondrex (USA). ELISA kits were from Thermo Fisher (Austria). Fluorescent dyes PKH26 and PKH67 kits were purchased from Beijing Baiao Laibo Technology; 1, 1'-Dioctadecyl-3, 3, 3', 3'-tetramethylindodicarbocyanine perchlorate (DID), 3-(4,5-dimethylthiazol-2-yl)-2,5-diphenyltetrazolium bromide (MTT), FITC-labeled phalloidin DAPI and Lipopolysaccharide (LPS) were supplied by Beijing Solarbio Science \& Technology. Other reagents were analytical grade.

\section{Cells and animals}

RAW 264.7 murine macrophages and human umbilical vein endothelial cells (HUVEC) (Chinese Academy of Sciences, Shanghai, China) were cultured in DMEM/ HIGH GLUCOSE medium (Hyclone, USA) with 10\% fetal bovine serum(FBS) (Gibco, USA) and 1\% (v/v) penicillin/streptomycin (Hyclone, USA).

Male DBA/1 mice (8 weeks old) were purchased from Charles River (Beijing, China). All animal experiments were in accordance with The Animal Ethics Committee of Southwest Medical University.

\section{Isolation and characterization of exosomes}

When RAW 264.7 cultures reached the logarithmic phase of growth, cells were switched to FBS-free medium for $24 \mathrm{~h}$, after which the medium was collected 
and replaced with fresh FBS-free medium. The cultures were incubated for another $24 \mathrm{~h}$, then the medium was collected again. The two volumes of collected medium were pooled, exosomes were isolated using a gradient centrifugation protocol [20] with some modification. Firstly, the medium was centrifuged at $2000 \mathrm{~g}$ for $10 \mathrm{~min}$ and then at 10,000 $\mathrm{g}$ for $30 \mathrm{~min}$ to remove cellular debris. Next, the supernatant was concentrated to about $30 \%$ of the original volume at $2000 \mathrm{~g}$ for $8 \mathrm{~min}$ using ultrafiltration tube $(\mathrm{MWCO}=10,000)$. Finally, the supernatant was centrifuged at 120,000 $g$ for $70 \mathrm{~min}$ in an ultracentrifuge (QPTimaMAX-XP Ultra-High, Beckman Coulter, USA). The pellets were washed with large volume cold phosphate-buffered saline (PBS) and centrifuged at 120,000 $\mathrm{g}$ for 70 min again to ensure maximal exosome purity. All centrifugation procedures were performed at $4{ }^{\circ} \mathrm{C}$. The pellet was re-suspended in PBS and stored at $-80{ }^{\circ} \mathrm{C}$. The amount of exosomes was estimated using a Bradford assay (Bio-Rad Laboratories, Shanghai, China).

Size, polydispersity index (PDI) and zeta potential of purified exosomes were determined using dynamic light scattering (Malvern Zetasizer Nano ZS90, Malvern Instruments, UK). Their morphology was examined using transmission electron microscopy (HT7700, Hitachi, Japan). The presence of CD63 and CD9 on the exosome surface were measured by western blotting. These proteins serve as markers of exosomes derived from mammalian cells $[18,26]$.

\section{Preparation of Exo/Dex and FPC-Exo/Dex}

To load Dex into exosomes, exosomes $(100 \mu \mathrm{g})$ were mixed with Dex $(300 \mu \mathrm{g})$ in PBS with the concentration of trehalose was $80 \mathrm{nM}$, which was added to avoid the aggregation of exosomes during electroporation. The mixture was subjected to electroporation at room temperature using a double poring pulse $(200 \mathrm{~V}, 5 \mathrm{~ms})$ and transfer pulse of five pulses $(20 \mathrm{~V}, 50 \mathrm{~ms})$ in a $1-\mathrm{cm}$ electroporation cuvette and a NEPA21 Type II electroporater (NEPA genes, Tokyo, Japan). Then un-encapsulated Dex was removed by ultracentrifugation $(100,000 \mathrm{~g}, 60 \mathrm{~min}$, $\left.4{ }^{\circ} \mathrm{C}\right)$. The Dex-loaded exosomes (Exo/Dex) were resuspended in PBS and incubated in $37^{\circ} \mathrm{C}$ for $1 \mathrm{~h}$ to restore the membrane.

The FA-PEG-Chol conjugate was prepared as described in Supplementary Materials. The Chol end of FA-PEGChol was inserted into the lipid bilayer membrane of Exo/ Dex by post-insertion [22]. Exo/Dex and FA-PEG-Chol ligands were mixed in a mass ratio of $1: 5$ and incubated at $37{ }^{\circ} \mathrm{C}$ for $2 \mathrm{~h}$. Free ligands were removed by centrifugation at $3000 \mathrm{~g}$ for $10 \mathrm{~min}$, giving rise to FPC-Exo/Dex. The amount of FA incorporated was determined by comparison of the $\mathrm{UV}_{285}$ value to a standard curve of folic $[27,28]$.

\section{Preparation of Dex-loaded anion liposomes (Lip/Dex) as control group}

Dex-loaded anionic liposomes were prepared in order to compare with exosome-based drug delivery systems. Anionic liposomes were prepared using ethanol injection as described [29]. Firstly, $40 \mathrm{mg}$ of yolk lecithin, $10 \mathrm{mg}$ of cholesterol and $10 \mathrm{mg}$ of Dex were dissolved in $3 \mathrm{~mL}$ ethanol, and the solution was slowly injected into $5 \mathrm{~mL}$ PBS (pH 7.4) while stirring. The solution was slowly stirred under bath conditions at $40{ }^{\circ} \mathrm{C}$ for $2 \mathrm{~h}$, and the solution was filtered through $0.45 \mu \mathrm{m}$ and $0.22 \mu \mathrm{m}$ membrane successively, yielding Lip/Dex.

\section{Characterization of different Dex preparations}

Size, PDI and Zeta potential of all preparations were determined using dynamic light scattering, and morphology of FPC-Exo/Dex were examined by transmission electron microscopy. Encapsulation efficiency (EE) and drug loading efficiency (DLE) were measured using highperformance liquid chromatography (HPLC). Briefly, each formulation was divided into two equal portions, one of which was demulsified with $10 \%$ methanol, and the amount of total drug $\left(\mathrm{W}_{\mathrm{t}}\right)$ was measured by HPLC. The free drug in the second portion $\left(\mathrm{W}_{\mathrm{e}}\right)$ was pelleted by ultracentrifugation and weighed. $\mathrm{EE}$ was calculated using the equation $\mathrm{EE}=\mathrm{W}_{\mathrm{e}} / \mathrm{W}_{\mathrm{t}} \times 100 \%$. EE of Lip/Dex was determined in the same way.

The amount of exosomes $\left(\mathrm{W}_{\mathrm{s}}\right)$ was estimated by $\mathrm{BCA}$ assay to calculate DLE according to the equation $\mathrm{DLE}=\mathrm{W}_{\mathrm{e}} /\left(\mathrm{W}_{\mathrm{t}}+\mathrm{W}_{\mathrm{s}}\right) \times 100 \%$. An equal amount of Lip/Dex was dried and weighed $\left(\mathrm{W}_{\mathrm{p}}\right)$ to calculate DLE according to the equation DLE $=\mathrm{W}_{\mathrm{e}} / \mathrm{W}_{\mathrm{p}} \times 100 \%$.

\section{In vitro cumulative drug release study}

FPC-Exo/Dex, Exo/Dex, Lip/Dex, or free Dex (40 $\mu \mathrm{g}$ of Dex contained in all preparations) were added to $1 \mathrm{~mL}$ PBS (pH 7.4 or pH 6.0) in a dialysis bag with a molecular weight cut-off of $3000 \mathrm{Da}$. The bag was placed in $30 \mathrm{~mL}$ PBS and shaken at $37^{\circ} \mathrm{C}$ at $1000 \mathrm{rpm}$. At predetermined time points, $200 \mu \mathrm{L}$ of release medium was collected and immediately replaced with an equal volume of fresh medium. Dex concentration was determined by HPLC, and the cumulative amount released was calculated.

\section{Toxicity assay of nanoparticles by MTT}

RAW264.7 cells and HUVEC in logarithmic growth phase were digested into single-cell suspensions and seeded in 96-well plates at $1 \times 10^{4}$ cells per well, then incubated at $37{ }^{\circ} \mathrm{C}$ overnight. FPC-Exo/Dex, Exo/Dex and Dex were prepared in culture medium without serum or antibiotics, and $200 \mu \mathrm{L}$ of each preparation was added to wells 
at Dex concentrations of $5-25 \mu \mathrm{g} / \mathrm{mL}$. After $24 \mathrm{~h}$, the medium was discarded, and $20 \mu \mathrm{L}$ of MTT $(5 \mathrm{mg} / \mathrm{mL})$ solution and $180 \mu \mathrm{L}$ of complete medium were added. Cells were cultured for another $4 \mathrm{~h}$, then medium was replaced with $150 \mu \mathrm{L}$ DMSO and cultures were shaken for $15 \mathrm{~min}$ at $37{ }^{\circ} \mathrm{C}$. Absorbance at $490 \mathrm{~nm}$ was measured using a Varioskan Flash microplate reader (Thermo Fisher, USA). Relative cell viability was calculated using the equation: Cell viability $=($ sample - blank $) /($ negative control - blank) $\times 100 \%$.

\section{Cellular uptake study by flow cytometry and confocal laser scanning microscopy}

Lip/Dex, Exo/Dex and FPC-Exo/Dex were labeled with PKH67 or PKH26 according to the dye manufacturer's protocol. PKH67-labeled Dex formulations were incubated for $2 \mathrm{~h}$ with resting or lipopolysaccharide (LPS)-activated (stimulated for $24 \mathrm{~h}$ with LPS at a final concentration of $100 \mathrm{ng} / \mathrm{mL}$ ) RAW264.7 cells. Uptake by cells was measured using a Verse cytometer (BD, USA).

In order to visually observe the situation of the formulations entering the cell, the three Dex formulations labeled by PKH26 were incubated with resting or LPSactivated RAW264.7 cells for $2 \mathrm{~h}$, then we analyzed endocytosis using confocal laser scanning microscopy (Leica SP8, Germany) after staining the cytoskeleton with FITCphalloidin and the nucleus with DAPI [18].

\section{Anti-inflammatory effects of nanoparticles to LPS-activated RAW264.7 cells}

RAW264.7 cells were seeded into a 24-well plate at a density of $5 \times 10^{5}$ cells per well and allowed to adhere, then stimulated for $24 \mathrm{~h}$ with LPS at a final concentration of $100 \mathrm{ng} / \mathrm{mL}$. The medium was replaced with fresh medium containing FPC-Exo/Dex, Exo/Dex, Lip/Dex or free Dex at a final Dex concentration of $20 \mu \mathrm{g} / \mathrm{mL}$. Negative control wells were incubated in culture contain PBS. After $24 \mathrm{~h}$, the culture medium was collected, centrifuged at $2000 \mathrm{~g}$ for $5 \mathrm{~min}$, and the supernatant was assayed for TNF- $\alpha$, IL- $\beta$ and IL-10 using ELISA kits according to the manufacturer's instructions [21].

\section{Establishing Mouse model of collagen-induced arthritis (CIA)}

Bovine type II collagen was thoroughly emulsified with an equal volume of complete Freund's adjuvant by vortex, and $100 \mu \mathrm{L}$ emulsion was administered intradermally at the base of the mouse tail. After 21 days, mice received an intradermal booster injection of type II collagen with an equal volume of incomplete Freund's adjuvant.
Biodistribution of nanoparticles in CIA mice

A total of 12 CIA mice were randomly divided into 4 groups (3 animals per group), which were intravenously administered DID-labeled Lip/Dex, Exo/Dex, FPCExo/Dex, or free DID (1 $\mu$ g DID per mouse). At $1,4,8$, and $24 \mathrm{~h}$ later, mice were anesthetized with $10 \%$ chloral hydrate $(0.04 \mathrm{~mL}$ per $10 \mathrm{~g})$ and analyzed using the IVIS ${ }^{\circledR}$ Spectrum system (Caliper, Hopkinton, MA, USA).

At $24 \mathrm{~h}$, mice were euthanized and the blood, heart, liver, spleen, lung, and kidney were removed. Blood was sampled and centrifuged at $3000 \mathrm{~g}$ for $7 \mathrm{~min}$ to obtain plasma. Fluorescence of plasma and organs was measured using the IVIS ${ }^{\circledR}$ Spectrum system (PerkinElmer, USA).

\section{Measurement of weight, paw thickness, foot volume and articular index (AI) score of CIA mice}

On day 21 after the booster immunization, CIA mice were randomly assigned to five groups (3 animals per group) and injected intravenously with free Dex, Lip/ Dex, Exo/Dex or FPC-Exo/Dex at a Dex dose of $1.2 \mathrm{mg} /$ $\mathrm{kg}$ in all cases. Negative control mice were injected with the same volume of Saline. The first injection was delivered on day 21, and then once every four days for a total of four injections. AI scores were determined for each limb as described [8]. Body weight, hind paw thickness and foot volume were measured every 3 days during treatment. Foot volume is measured with drainage method.

\section{Micro-computed tomography (Micro-CT) analyses of articular bone}

After mice were sacrificed, the left hind limbs were removed and immediately fixed in $4 \%$ paraformaldehyde for $48 \mathrm{~h}$. Then the microstructure of each limb was analyzed using a SIEMENS Inveon PET/CT computed tomography system (SIEMENS, Germany) with the following parameters: voltage, $80 \mathrm{kV}$; current, $500 \mu \mathrm{A}$; exposure time, $1800 \mathrm{~ms}$; total rotation, $220^{\circ}$; and projections, 120 sheets. A region of interest (ROI) of the trabecular bone within the calcaneus was defined by aligning the calcaneus bone along the sagittal plane using the Data Viewer, starting $0.2 \mathrm{~mm}$ away from the epiphyseal plate and continuing for $40-50$ slides $(1 \mathrm{~mm})$ [30, 31]. The bone mineral density (BMD), percent bone volume (BV/ TV), bone surface density (BS/BV), trabecular thickness (Tb.Th), trabecular number (Tb.N) and trabecular spacing (Tb.Sp) of the ROI were calculated using SIEMENS Inveon Research Workplace software 4.2.

\section{Histological evaluation of joint tissues}

Ankle joints were dissected from each group and fixed in $4 \%$ paraformaldehyde for $48 \mathrm{~h}$, and then decalcified in $10 \%$ neutral EDTA solution for 15 days at room temperature. 
Then decalcified tissue was embedded in paraffin. Thin Sects. $(5 \mu \mathrm{m})$ were cut and stained with hematoxylineosin $(\mathrm{H} \& \mathrm{E})$ or safranin $\mathrm{O}(\mathrm{SO})$ combines with chondroitin sulfate to stain articular cartilage red [32]. An H\&E score from 0 to 3 was determined for each of the following aspects: inflammatory cell infiltration, synovial tissue proliferation, fibrous tissue hyperplasia, and macrophage infiltration. Then all these scores were summed for a given ankle joint, and the overall scores for all ankle joints were summed to obtain H\&E scores for a given animal.

\section{Evaluation of inflammatory cytokines in serum}

Blood samples were collected from mice on day 45 after induction of CIA. Serum levels of inflammatory cytokines TNF- $\alpha$, IL- $1 \beta$ and IL-10 were measured using ELISA kits according to the manufacturer's instructions.

\section{In vivo safety evaluation of nanoparticles in CIA mice}

Aspartate transaminase (AST) and alanine transaminase (ALT) levels of serum collecting from different treatment groups were assayed using a commercial kit (Nanjing Institute of Biological Engineering, Nanjing, China) according to the manufacturer's protocol.

\section{Statistical analysis}

Statistical analysis was performed using GraphPad Prism 6.0 (GraphPad Software, La Jolla, CA, USA). Statistical comparisons were performed by one-way ANOVA (Dunnett's multiple comparisons test) for multiple groups, except for the analyses in Figs. $4 \mathrm{~b}$ and $6 \mathrm{~b}$, which were performed using two-way ANOVA. Differences associated with $p<0.05$ were considered significant. All results were expressed as mean $\pm \mathrm{SD}$.

\section{Results}

\section{Characterization of exosomes}

Exosomes (Exos) showed an average size of $98.87 \pm 6.69 \mathrm{~nm}$, PDI of $0.36 \pm 0.01$, and zeta potential of $-12.03 \pm 1.47 \mathrm{mV}$ (Fig. 1a, b). Consistent with these results, transmission electron microscopy displayed that exosomes were round-shaped nanovesicles surrounded by membrane, with a diameter of approximately $100 \mathrm{~nm}$ (Fig. 1c). Ultimately, $2.20 \pm 0.44 \mu \mathrm{g}$ of exosomes (based on protein content) could be purified from $1 \mathrm{~mL}$ of culture medium. Western blot experiments showed that the exosomes contained the marker proteins CD9 and CD63 (Fig. 1d).

\section{Structure of FA-NHS and FA-PEG-Chol}

${ }^{1} \mathrm{H}-\mathrm{NMR}$ showed signals at $6.93 \mathrm{ppm}$ and $7.60 \mathrm{ppm}$ belonging to the para-aminobenzoic acid moiety in FA, as well as signals at 4.21-4.30, 1.79-2.05 and
2.20-2.31 ppm belonging to the $\alpha-, \beta-$, and $\gamma-\mathrm{CH}_{2}-$ protons of the glutamic acid moiety of FA (Additional file 1: Fig. S1). The signal at 2.44-2.49 ppm was attributed to the proton of the NHS group.

${ }^{1} \mathrm{H}-\mathrm{NMR}$ of FA-PEG-Chol confirmed the formation of the conjugate, showing principal peaks related to FA (8.53-8.78, 7.73-7.65, 5.60-5.52, 4.64-4.53 ppm), the PEG moiety (3.46-3.64 ppm) and the Chol moiety (0.860.82, 0.62-0.69 ppm) (Additional file 1: Fig. S2).

\section{Characterization of different Dex preparations and incorporation of FA-PEG-Chol into Exo/Dex} Average diameter was smallest for Exo/Dex $(106.27 \pm 11.40 \mathrm{~nm})$, intermediate for Lip/Dex $(112.60 \pm 10.61 \mathrm{~nm})$ and largest for FPC-Exo/Dex $(128.43 \pm 16.27 \mathrm{~nm})$ (Fig. 2a-d). Nevertheless, the three formulations did not differ significantly in size. Zeta potential was $-34.80 \pm 6.60 \mathrm{mV}$ for Exo/Dex, $-11.00 \pm 0.61 \mathrm{mV}$ for Lip/Dex and $-22.73 \pm 0.91 \mathrm{mV}$ for FPC-Exo/Dex (Fig. 2a). PDI was approximately 0.3 for all three formulations, indicating narrow size distribution. Transmission electron microscopy showed that Exo/Dex, FPC-Exo/Dex and Lip/Dex all exhibited typical sphere-like shapes (Fig. 2e-g). We used UV spectroscopy to determine that $21.37 \pm 5.46 \mu \mathrm{g}$ of FA was incorporated into $100 \mu \mathrm{g}$ of exosomes.

Exo/Dex showed EE of $11.12 \pm 1.82 \%$ and DLE of $22.38 \pm 1.13 \%$. The corresponding values for FPC-Exo/ Dex were lower $(10.26 \pm 0.73 \%$ and $18.81 \pm 2.05 \%)$, as were the values for Lip/Dex $(83.36 \pm 1.43 \%$ and $13.54 \pm 2.09 \%$ ) (Fig. 2a).

\section{In vitro cumulative drug release and toxicity of nanoparticles}

Dex was released from FPC-Exo/Dex in a slow, sustained fashion at $\mathrm{pH} 7.4$ (Fig. 3a). During the first $8 \mathrm{~h}$, only $47.26 \%$ of Dex was released from FPC-Exo/Dex, compared to $90.46 \%$ of Dex from the free drug solution. Drug release from Exo/Dex and Lip/Dex was intermediate between these two extremes. Under acidic conditions of $\mathrm{pH} 6.0$, nearly $80 \%$ of encapsulated drug from FPC-Exo/Dex was released by $16 \mathrm{~h}$ (Fig. 3b). However, less than $60 \%$ released within $16 \mathrm{~h}$ at $\mathrm{pH} 7.4$ (Fig. 3a), suggesting acidic $\mathrm{pH}$ triggered faster drug release from preparations.

Exposing HUVEC cultures for $24 \mathrm{~h}$ to free Dex or any of the Dex-loading formulations had no obvious effect on viability, which remained above $80 \%$ even at a concentration of $25 \mu \mathrm{g} / \mathrm{mL}$ (Fig. 3c). When we performed these toxicity experiments on RAW264.7 cultures, we found no significant decrease in viability with FPC-Exo/Dex, and a slight decrease with free Dex (Fig. 3d). 

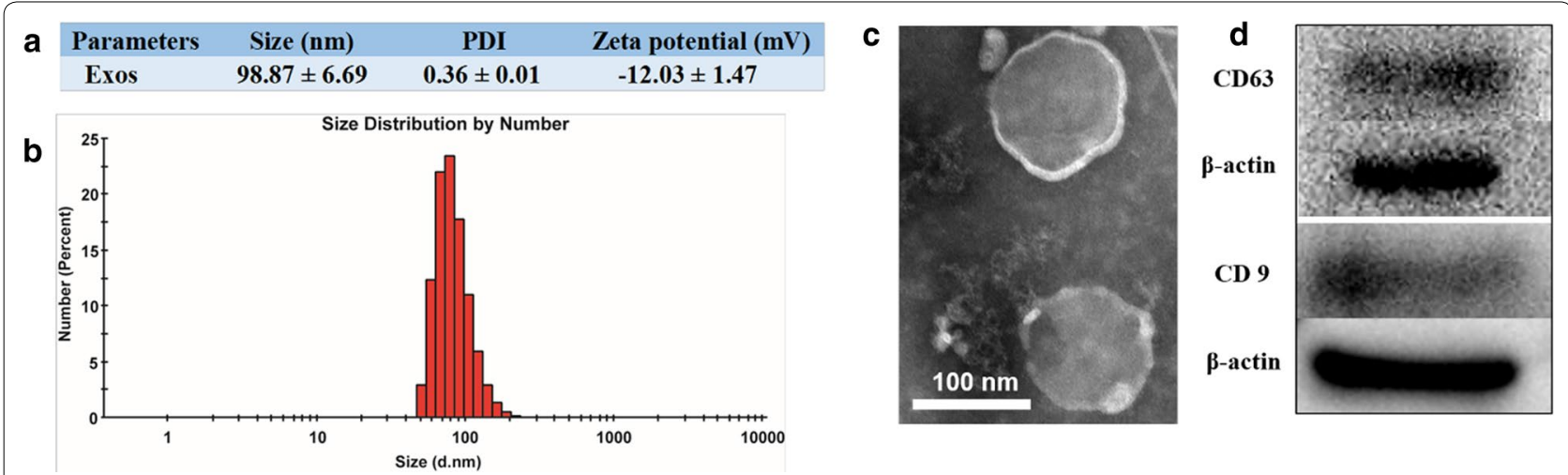

Fig. 1 Characterization of exosomes. a Size, PDI and zeta potential of Exos. b Size distribution of Exos. c Transmission electron micrographs of Exos. e Western blot to confirm the expression of CD9 and CD63

\section{Cellular uptake of nanoparticles by RAW264.7 cells}

RAW264.7 cells internalized larger amounts of Dex formulations after activation with LPS, especially the largest of FPC-Exo/Dex (Fig. 4a, b). Regardless of activation status, cells took up more FPC-Exo/Dex than the other Dex formulations. Moreover, the endocytosis of Exo/Dex was more than Lip/Dex. These flow cytometry results were supported by confocal imaging (Fig. 4c, d). Confocal images instruct that FPC-Exo/Dex entered the cytoplasm the most, followed by Exo/Dex, especially in LPS-activated RAW264.7 cells.

\section{Effect to inflammatory cytokines secretion}

TNF- $\alpha$ levels in culture medium were much lower after LPS-activated RAW264.7 cells were treated with FPCExo/Dex and Exo/Dex than with free Dex or Lip/Dex (Fig. 5a). Similarly, the formulations inhibited secretion of IL-1 $\beta$, with FPC-Exo/Dex showing a stronger effect, then followed by Exo/Dex (Fig. 5b), suggesting that the exosome-based drug delivery system exerts better antiinflammatory effects. FPC-Exo/Dex significantly up-regulated the anti-inflammatory cytokine IL-10 $(p<0.01)$, Exo/Dex also did $(p<0.05)$ (Fig. 5c).

\section{Biodistribution of nanoparticles in CIA mice}

Real-time fluorescence imaging revealed that all three DIDlabeled preparations accumulated in the joints of CIA mice at $1 \mathrm{~h}$ (Fig. 6a), presumably due to the ELVIS effect. DID/ FPC-Exo/Dex showed the greatest accumulation into joints at every time point. Even at $24 \mathrm{~h}$, the DID/FPC-Exo/Dex group showed intensive fluorescence, whereas the DID/ Lip/Dex group showed slight signal by $8 \mathrm{~h}$ (Fig. 6a). Semiquantitation of fluorescence intensity in ankle joints further indicate that DID/FPC-Exo/Dex group displayed more significant fluorescence than other groups (Fig. 6c). In addition,
DID/Exo/Dex group displayed significantly fluorescence at 4, 8 and 24 h compare to DID/Lip/Dex group (Fig. 6c). These results suggest that FPC-Exo/Dex may target inflammatory lesions better and persist there longer than Exo/Dex and Lip/Dex, and Exo/Dex showed better targeting performance than Lip/Dex. Ex vivo imaging indicated that DID/FPC-Exo/ Dex accumulated more in plasma than other groups after $24 \mathrm{~h}$, suggesting its long systemic circulation (Fig. 6b, d). Furthermore, obvious fluorescence was detected in livers of all groups, however, DID/FPC-Exo/Dex group had lower distribution than Exo/Dex (Fig. 6b, d), suggesting modifying with FPC could decrease non-specific distribution. Fluorescence in spleens and lungs was faint, and almost no fluorescence was detected in kidney (Fig. 6b, d).

\section{Therapeutic efficacy in CIA mice based on body weight, Al scores, paw thickness and foot volume}

Body weight serves as an indirect indicator of therapeutic efficacy in RA since disease progression is associated with weight loss due to less feeding and greater apathy [33]. Body weight in mice injected with FPC-Exo/Dex increased continuously from day 21 (2nd booster injection) until day 45 , and it had no significant difference with Normal group (Fig. 7a). All treatment groups showed an initial increase in scores, reflecting disease progression, followed by a decrease in scores, reflecting therapeutic effects (Fig. 7b). FPC-Exo/Dex was associated with the lowest AI scores (Fig. 7b). This formulation also led to the least swelling of the hind limbs, based on paw thickness and foot volume, followed by Exo/Dex (Fig. 7c, d).

Imaging of the left hind limbs in animals treated with saline showed severe, extensive joint swelling and joint deformation, which free Dex reduced slightly but not markedly (Fig. 7e). FPC-Exo/Dex reduced this swelling nearly completely, such that the difference from saline-treated animals was not significant. Exo/Dex also 


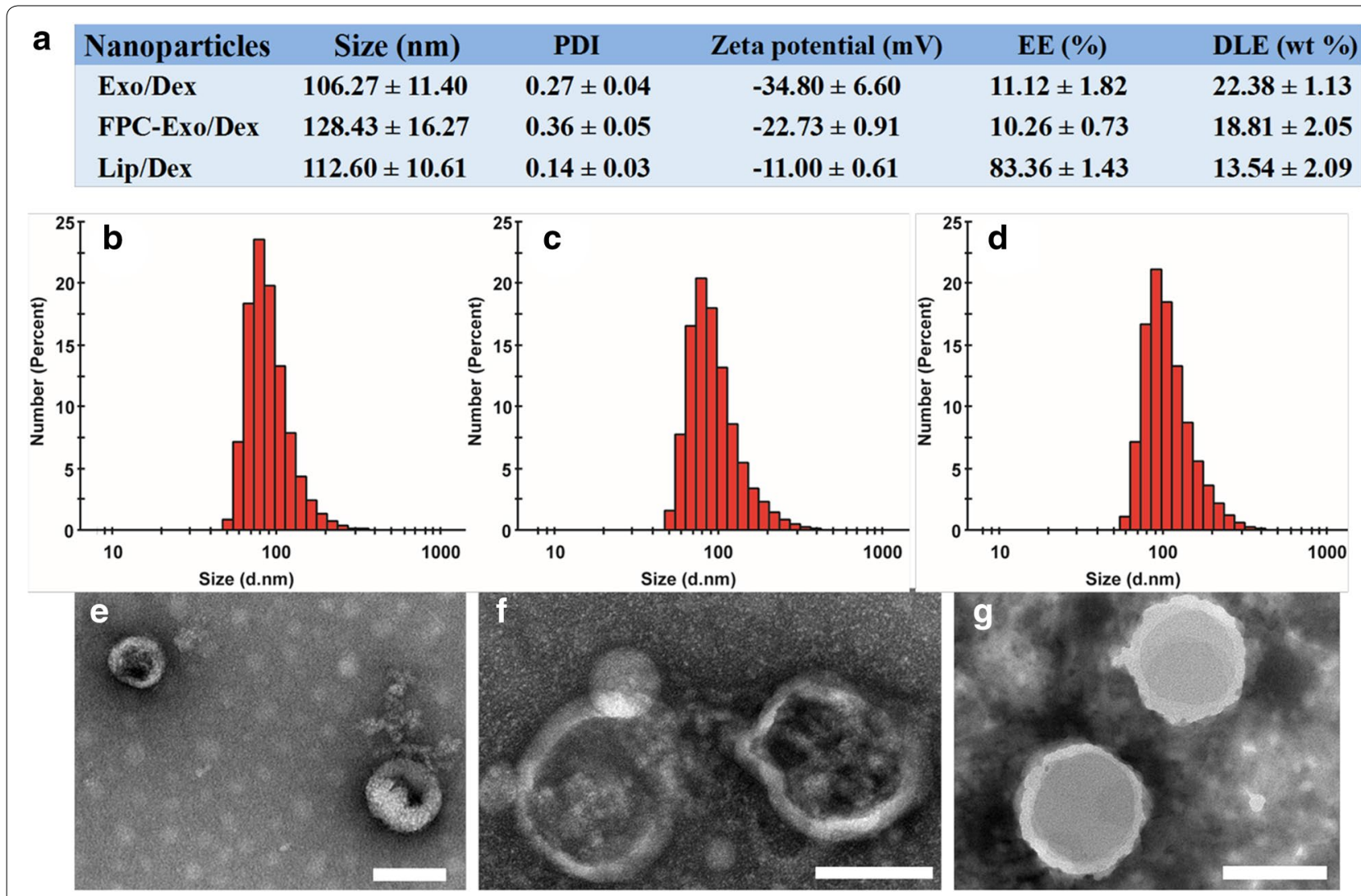

Fig. 2 Characterization of Exo/Dex, FPC-Exo/Dex and Lip/Dex. a Size, PDI, zeta potential, EE and DLE of Dex preparations. Size distribution of Exo/ Dex b, FPC-Exo/Dex c and Lip/Dex $\mathbf{d}$. Transmission electron micrographs of Exo/Dex e, FPC-Exo/Dex $\mathbf{f}$ and Lip/Dex $\mathbf{g}$
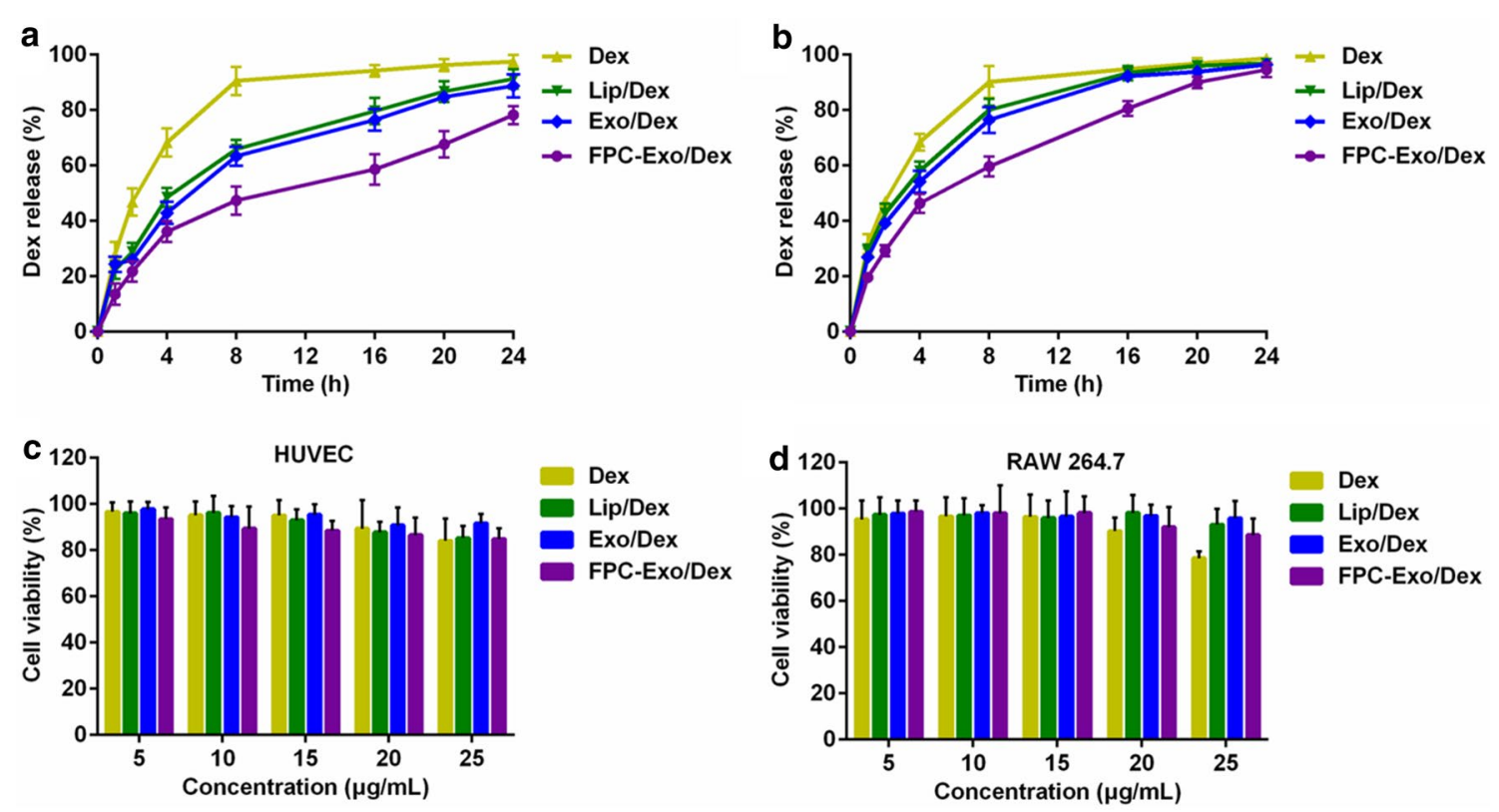

Fig. 3 In vitro cumulative release and toxicity of Dex preparations. Cumulative release of Dex from Lip/Dex, Exo/Dex and FPC-Exo/Dex at PBS of pH $7.4 \mathbf{a}$ or pH 6.0 b. Results were shown as mean \pm SD $(n=3)$. The effect of Dex, Lip/Dex, Exo/Dex and FPC-Exo/Dex for HUVEC c and RAW264.7 cells viability $\mathbf{d}$. Data represent mean $\pm S D(n=5)$ 


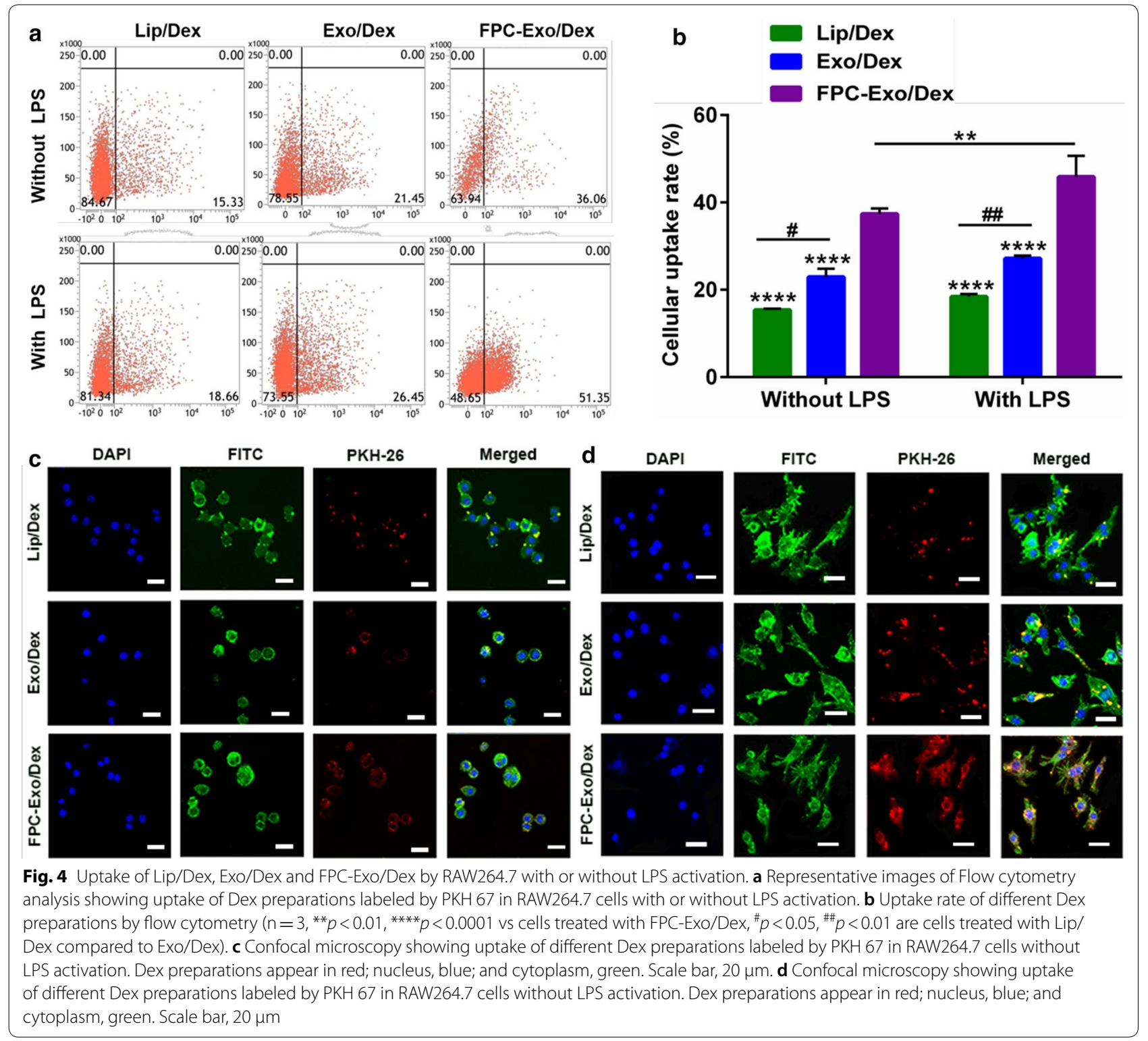

significantly ameliorated swelling, but not as much as FPC-Exo/Dex.

\section{Microstructure of articular bone based on Micro-CT}

Saline-treated animals showed the most severe damage, with extensive erosion of the bone in ankle and toe joints (Fig. 8a). FPC-Exo/Dex was associated with significantly lower ankle bone erosion, which was no longer obvious after four times administrations. Quantitative analysis of the ROI in calcaneus showed that FPC-Exo/Dex treatment preserved the bone quality as evident in the morphometric parameters, such as bone mineral density (BMD), percent bone volume (BV/TV), bone surface density (BS/BV), trabecular thickness (Tb.Th), trabecular number (Tb.N) and trabecular spacing (Tb.Sp), with their values similar to those observed for healthy controls; significantly better than those observed for other Dex formulations treated groups (Fig. 8b-g). Meanwhile, the values of BMD, BV/TV, BS/BV and Tb.Th in Exo/ Dex-treated group are significantly lower than these of Lip/Dex-treated groups, and Exo/Dex-treated group displayed a slightly lower articular bone erosion.

\section{Histological analysis of joint tissue}

Compared to healthy control animals, H\&E staining of histology slices from CIA mice treated with saline or free Dex revealed marked inflammatory cell infiltration, synovial tissue expansion and fibrous tissue hyperplasia (Fig. 9a). Average histopathology scores were 7.0 in the Saline group and 6.0 in the Dex-treated group. Animals 

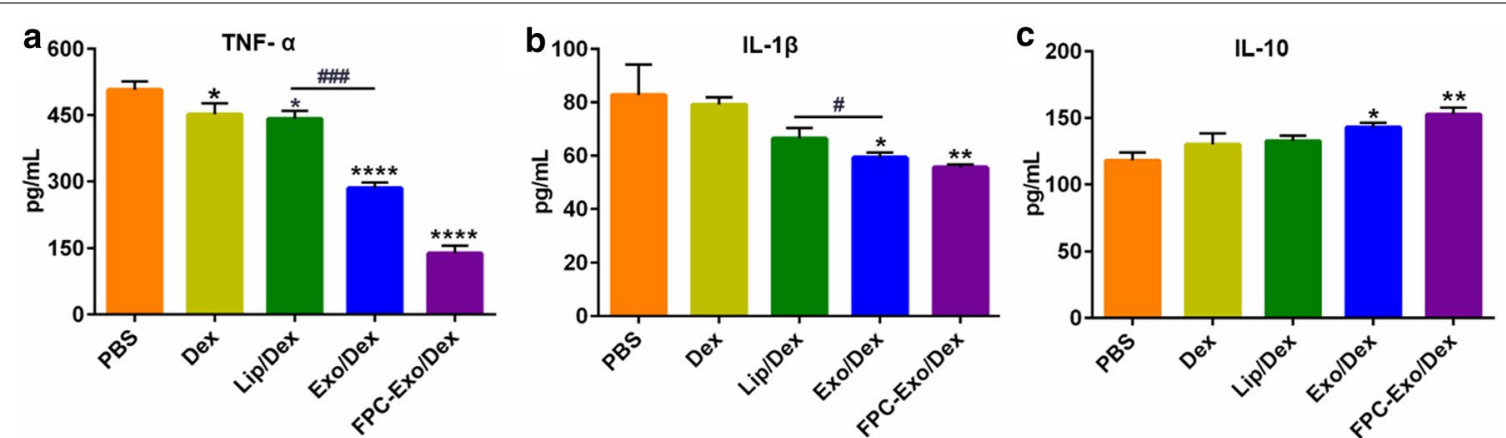

Fig. 5 Expression of inflammatory cytokines in cell-free supernatants treated with different preparations. Level of a TNF-a, $\mathbf{b} \| L-1 \beta$, and $\mathbf{c} \| L-10(n=3$, ${ }^{*} p<0.05,{ }^{* *} p<0.01,{ }^{* * *} p<0.001$ vs cells treated with PBS, ${ }^{\#} p<0.05,{ }^{\# \# \#} p<0.001$ are cells treated with Lip/Dex compared to Exo/Dex)

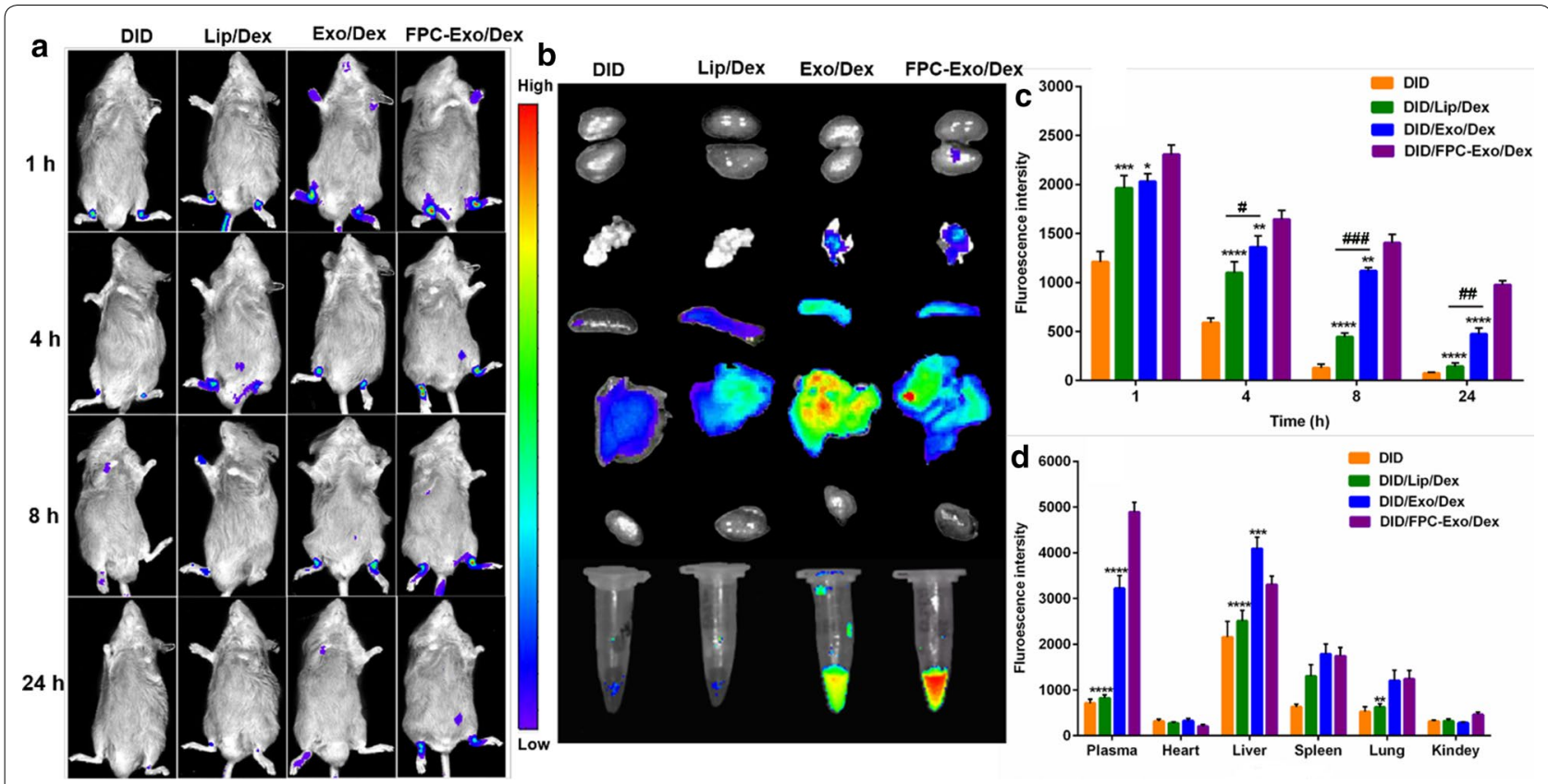

Fig. 6 The real-time fluorescence imaging of CIA mice. a Real-time fluorescence imaging of CIA mice after intravenous injection with free DID, DID/ Lip/Dex, DID/Exo/Dex and DID/FPC-Exo/Dex, respectively $(n=3)$. b Ex vivo imaging of the plasma and organs at $24 \mathrm{~h}$ after intravenous injection. $\mathbf{c}$ Semi-quantitation of fluorescence intensity in joints. $\mathbf{d}$ Semi-quantitation of fluorescence intensity in joints in plasma and organs $\left(n=3,{ }^{*} p<0.05\right.$, ${ }^{* *} p<0.01,{ }^{* * *} p<0.001,{ }^{* * *} p<0.0001$ vs mice injectedted with DID/FPC-Exo/Dex, ${ }^{*} p<0.05,{ }^{\# \#} p<0.01,{ }^{\# \#} p<0.001$ are mice injected with DID/Lip/ Dex compared to DID/Exo/Dex)

treated with Lip/Dex showed a score of 4.3, indicating moderate damage. The corresponding scores in the Exo/Dex and FPC-Exo/Dex groups were 2.3 and 0.7, indicating markedly reduced joint damage of FPC-Exo/ Dex (Fig. 9c).

Based on SO staining, articular cartilage was nearly destroyed in animals treated with saline or free Dex (Fig. 9b), but the destruction was significantly less severe in animals treated with FPC-Exo/Dex. In addition, joints from animals treated with Lip/Dex showed weaker red staining on articular surfaces than Exo/
Dex-treated animals, suggesting severer degradation and destruction of articular cartilage tissue. Qualitative analysis suggested that FPC-Exo/Dex protected the cartilage to the greatest extent.

\section{Inflammatory Cytokines in serum of $\mathrm{ClA}$ mice} after treatment

Following CIA induction, levels of both TNF- $\alpha$ and $\mathrm{IL}-1 \beta$ in the serum increased, and these increases were marginally but not significantly smaller with free Dex or Lip/Dex, while significantly smaller with FPC-Exo/ 
Dex $(p<0.01)$ and Exo/Dex $(p<0.05)$ were observed (Fig. 10a, b). In addition, the level of IL-1 $\beta$ in Exo/Dextreated group is lower than it of Lip/Dex-treated group. Meanwhile, FPC-Exo/Dex treatment increased the level of IL-10 the most in all groups $(p<0.001)$, then Exo/Dex $(p<0.01)$ (Fig. 10c).

\section{Hepatotoxicity analysis of nanoparticles in CIA mice after treatment}

Serum levels of AST and ALT in any of the Dex-treated groups were not significantly different from those in the healthy, untreated controls (Fig. 11), indicating that this therapeutic strategy may not cause obvious damage to the liver. FPC-Exo/Dex was associated with marginally but not significantly levels of both indices than free Dex (Fig. 11). These results suggest desirable biocompatibility of FPC-Exo/Dex in vivo.

\section{Discussion}

In this study, we derived the exosomes from RAW264.7 macrophages because inflammatory lesions in RA contain abundant activated macrophages, and target cells appear to internalize exosomes better when these exosomes have been derived from the same cell type
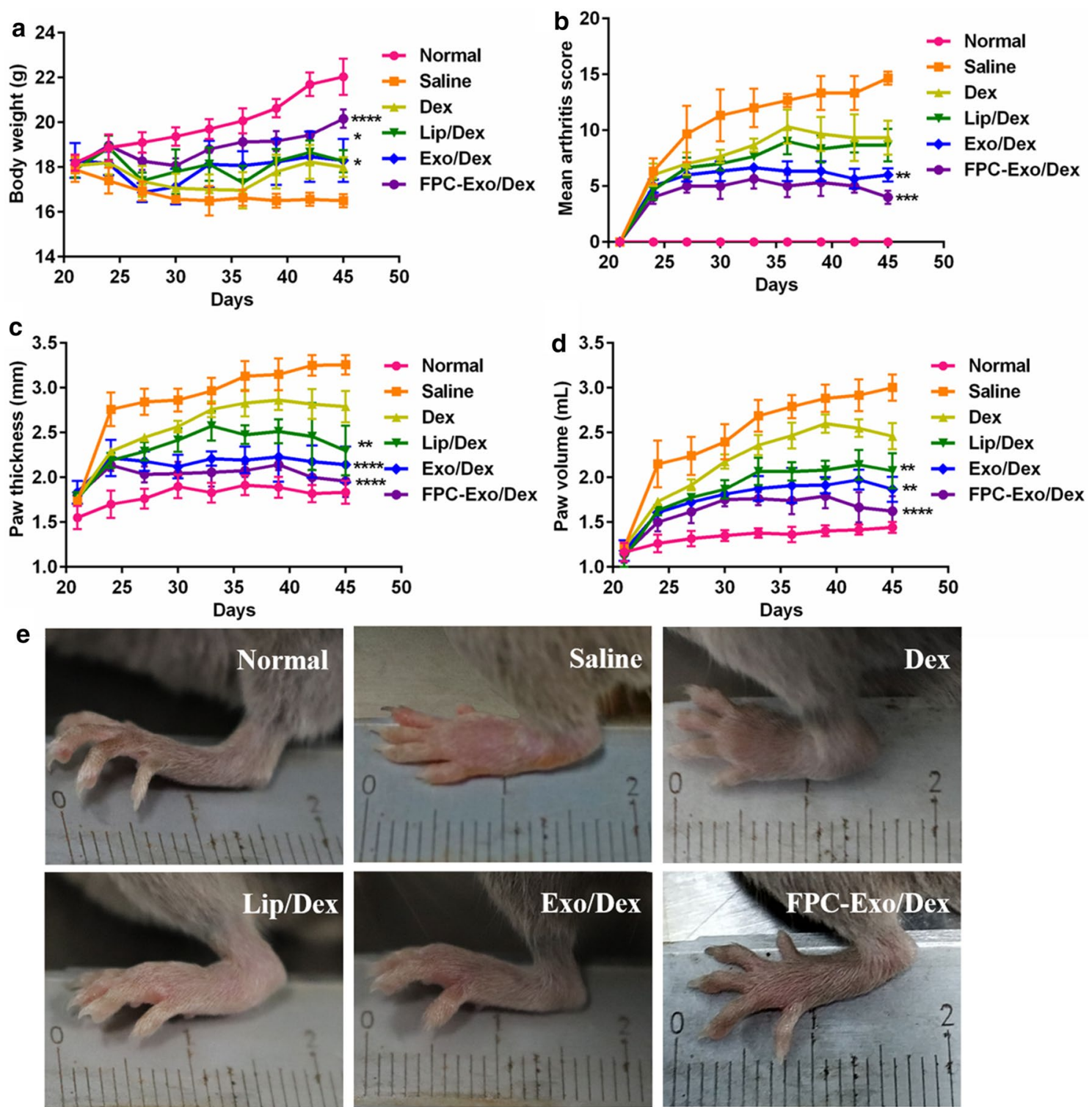

Fig. 7 Therapeutic indicators monitoring during treatment. Measurement of body weight $\mathbf{a}$, articular score $\mathbf{b}$, paw thicknessc, paw volume $\mathbf{d}$ and photographs of representative hind limbs from animals treated with various Dex formulations in $\mathrm{CIA}$ mice $\mathbf{e}$. (n=3, ${ }^{*} p<0.05,{ }^{* *} p<0.01,{ }^{* * *} p<0.001$, **** $p<0.0001$ vs mice injected with Saline) 
[26, 34-36]. Furthermore, it was reported that exosomes derived from macrophages have achieved excellent therapeutic efficacy in the treatment of other inflammations [20, 22]. Therefore, the choice of macrophages as the donor cell of exosomes is cogitative. We extract exosomes using ultracentrifugation because this classical approach may extract exosomes more efficiently and provide better dispersity than other extraction methods $[37,38]$. We substantially shortened the ultracentrifugation time by adding an initial ultrafiltration to reduce the volume of the medium before the second ultracentrifugation. Dynamic light scattering showed the resulting exosomes to be uniformly disperse in the narrow range of $80-110 \mathrm{~nm}$, and a protein assay estimated a yield of
$2.20 \pm 0.44 \mu \mathrm{g}$ of exosomes from $1 \mathrm{~mL}$ of culture medium. These results indicate that our combination of ultrafiltration and ultracentrifugation is suitable for the extraction of exosomes from RAW264.7 cells with adequate yields for subsequent experiments.

Electroporation is widely used to load chemical drug, short interfering RNA and DNA into exosomes [39, 40]. We included $80 \mathrm{mM}$ trehalose in the mixture of Dex and exosomes during electroporation to prevent aggregation of the exosomes [41], which may help explain why we obtained a uniform dispersion of size with narrow PDI.

We modified the exosomes with FA-PEG-Chol compound (FPC) to optimize their properties for better treating of RA, for folic acid can help the particles

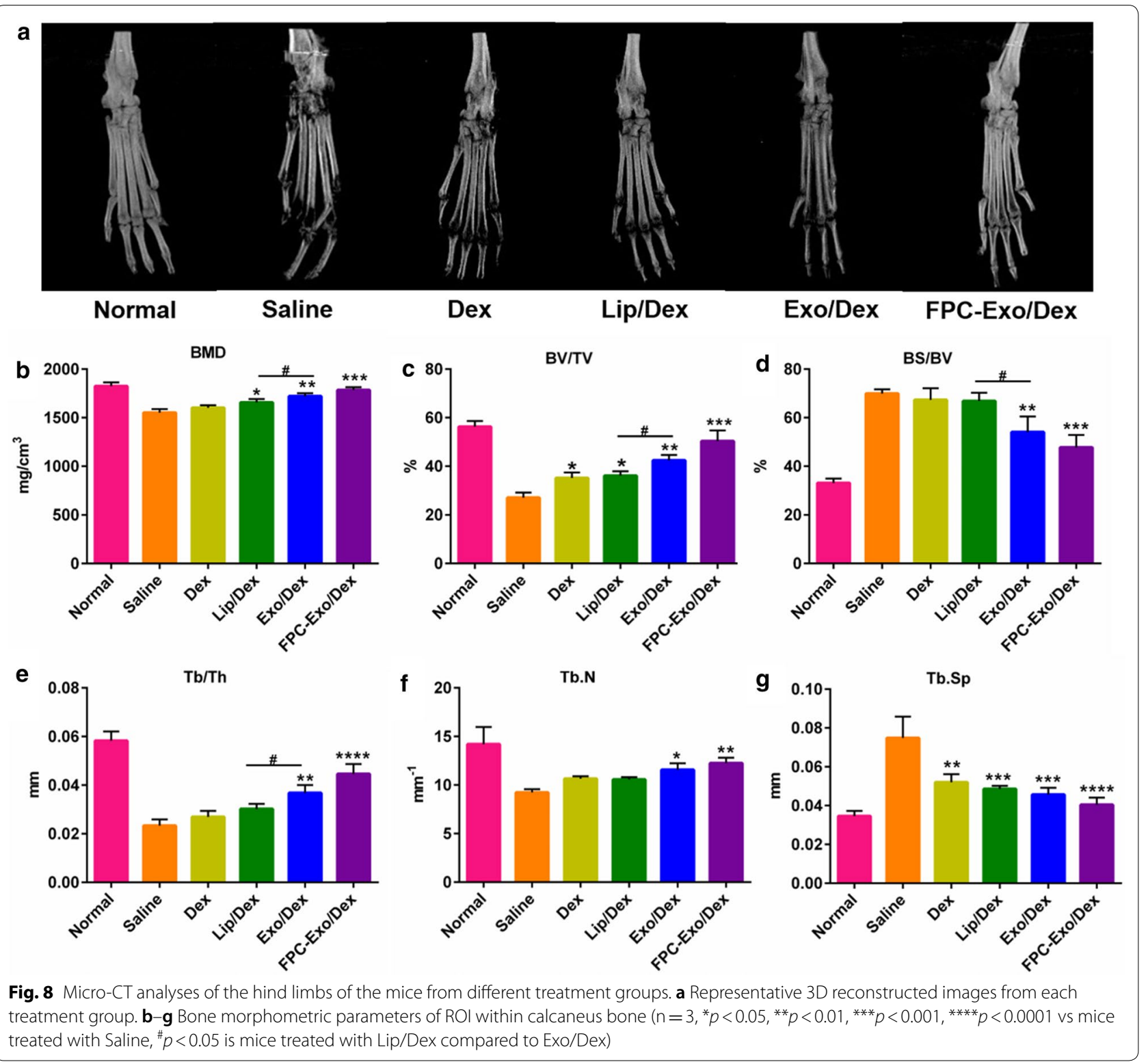




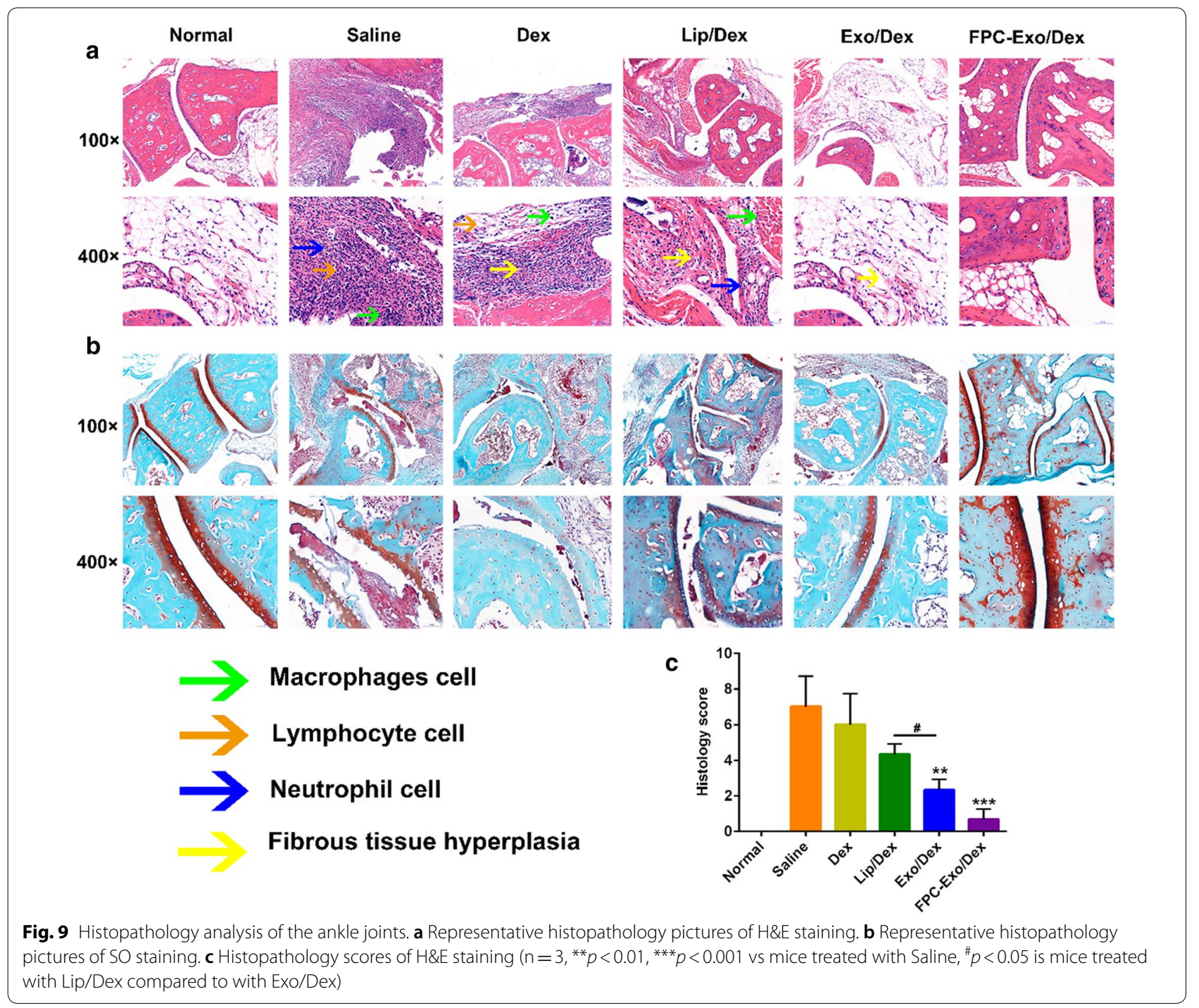

accumulate and persist in inflamed tissues, where abundant activated macrophages express FR [3]. Moreover, PEG can enhance stability and long circulation time of drug delivery system [42], and both the PEG and Chol help solubilize the FA and ensure its stable incorporation into the membrane of the exosomes. In vitro cumulative drug release study indicated that Dex was released more sustainably from FPC-Exo/Dex than from Exo/Dex or free Dex. Biodistribution examination further showed that FPC-Exo/Dex was retained more and longer in joint tissues than Exo/Dex in CIA mice. These results confirm that our "FPC" modification of Dex-loaded exosomes ensured their active targeting to inflammatory sites in RA, but also due to the fact that PEG can prolong circulation time. Consistent with this, cellular uptake studies also indicted that FPC-Exo/Dex was taken up by LPS-activated macrophages to a greater extent than Exo/Dex or Lip/Dex.

Dex and other glucocorticoids are used to treat RA because they down-regulate secretion of pro-inflammatory cytokines such as IL-1 $\beta$, TNF- $\alpha$ and IL- 6 by activated macrophages at inflammation lesions, while upregulating secretion of the anti-inflammatory cytokine IL-10 [10, 11, 19]. We found that FPC-Exo/Dex triggered these therapeutic effects in CIA mice to a significantly greater extent than other Dex formulations. These effects correlated with significantly lower histopathology scores based on H\&E staining analysis, significantly less cartilage and articular bone destruction. based on SO staining and micro-computed tomography analysis. The less severe bone destruction may be an indirect result of the ability of FPC-Exo/Dex to reduce secretion of IL-1 $\beta$ 

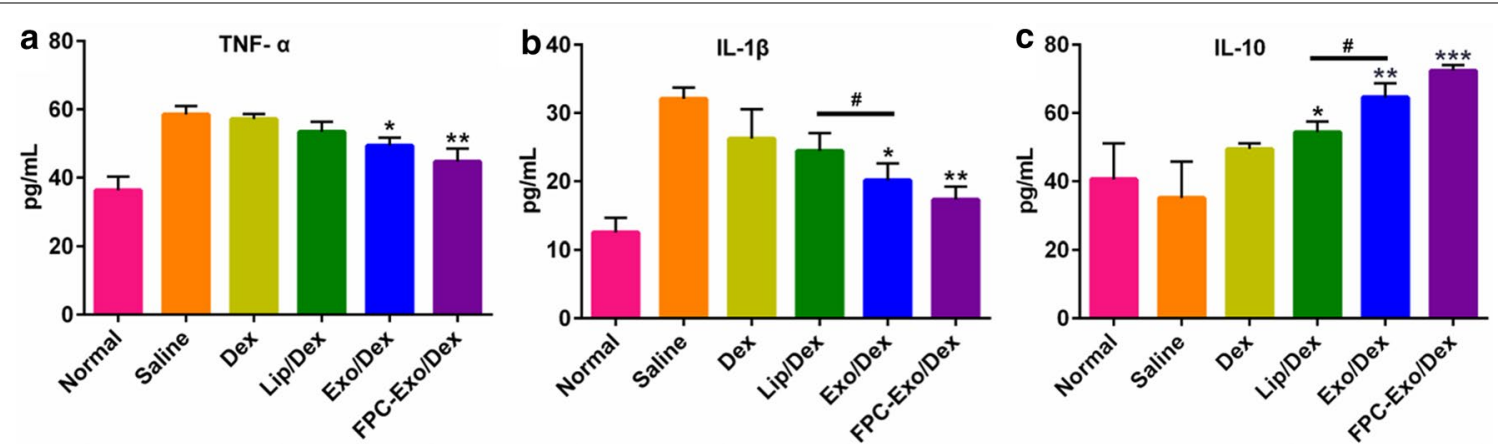

Fig. 10 Levels of the inflammatory cytokines in serum. Level of TNF- $a \mathbf{a}, \mathrm{IL}-1 \beta \mathbf{b}$, and $\mathrm{IL}-10 \mathbf{c}$, results are shown as mean $\pm S D\left(n=3,{ }^{*} p<0.05\right.$, ${ }^{* *} p<0.01,{ }^{* * *} p<0.001$ vs mice treated with Saline, ${ }^{*} p<0.05$ is mice treated with Lip/Dex compared to Exo/Dex)

and TNF- $\alpha$, since these factors may promote the formation of osteoclasts [43-45]; and to promote secretion of IL-10, which can inhibit osteoclast formation. The superior therapeutic effects of FPC-Exo/Dex over the other Dex formulations may relate to its better targeting and internalization.

FPC-Exo/Dex showed good biocompatibility not only in culture studies with HUVEC and RAW264.7 cells but also in mice. Exosomes tend to accumulate in the liver after intravenous administration [46, 47], and biodistribution assay showed substantial accumulation of Exo/ Dex and FPC-Exo/Dex in liver. However, there was no significant difference between Exo/Dex or FPC-Exo/Dex group and normal group for the liver function indices AST and ALT, suggesting that encapsulating Dex into exosomes did not cause hepatotoxicity.

Results of comparative research between Exo/Dex and Lip/Dex suggest that exosome-based on drug delivery system had better internalization, weaker blood clearance and long-term circulating capability. Consistently, Exo/Dex displayed better therapeutic efficacy than Lip/
Dex, reflecting the advantages of exosome as an endogenous nanocarrier over liposome.

Although exosome is an idea nanocarrier to delivery GCs for treatment to RA, the encapsulation efficiency of exosome-based drug system is mostly less than $30 \%$ $[22,48]$, which is much lower than the encapsulation efficiency in physical nanoparticles (almost over 90\%) $[8,49]$. It has been reported that encapsulating drugloading physical nanoparticles into exosomes could enhance the encapsulation efficiency of drug delivery system and take endogenous advantage of exosomes $[50,51]$, which also provides new idea for our future research.

\section{Conclusion}

We prepared relatively simple, biocompatible FPC-Exo/ Dex nanoparticles as a drug delivery platform. These particles show the advantages of other formulations as well as greater stability and longer persistence because of their PEG-Chol-FA modification. These modified
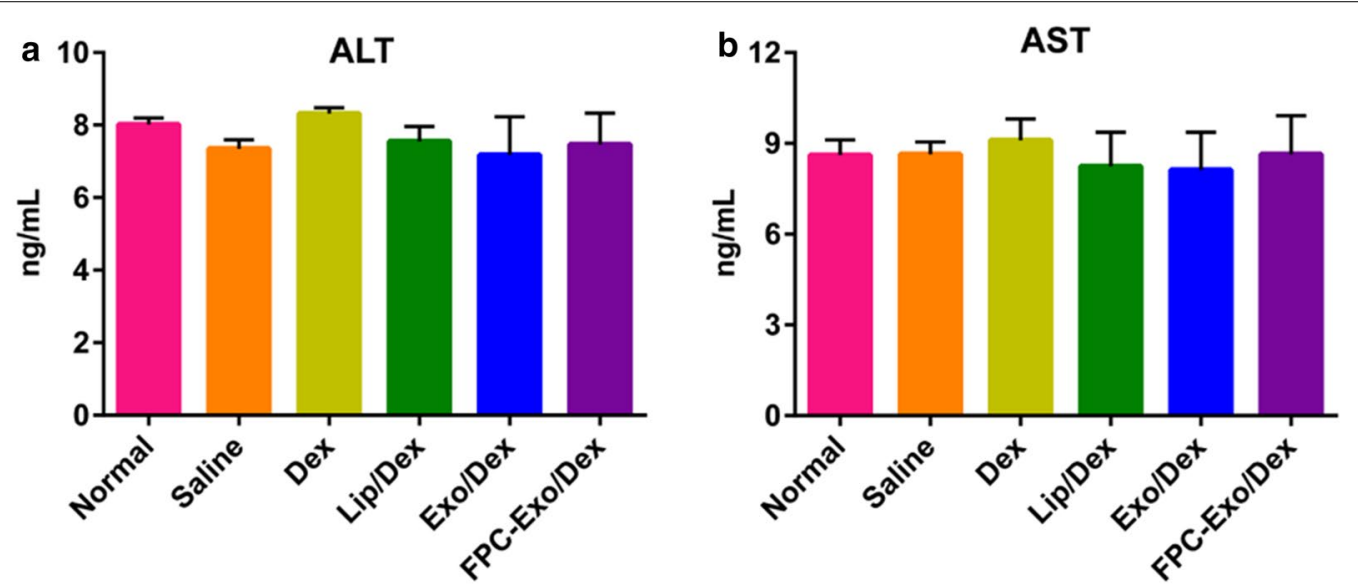

Fig. 11 Levels of AST and ALT in serum $(n=3)$. All the treatment groups were compared to Normal group 
exosomes were endocytosed better in vitro than Exo/ Dex, or free Dex, and they targeted inflamed joints and protected bone and cartilage better in mice with collagen-induced arthritis. They down-regulated the levels of pro-inflammatory cytokines and up-regulated antiinflammatory cytokine, strongly inhibiting macrophagedriven inflammation in CIA mice. The FPC-Exo/Dex system constructed in our study may be a useful drug delivery system for GCs to treat RA.

\section{Supplementary information}

Supplementary information accompanies this paper at https://doi. org/10.1186/s12951-020-00675-6.

Additional file 1. Fig. S1. 1 H NMR spectra of FA-NHS. Fig. S2. 1 H NMR spectra of FA-PEG-Chol. Fig. S3. The formation of FA-NHS ester. Fig. S4. The reaction scheme of FA-NHS with Chol-PEG-NH2.

\section{Abbreviations}

GCs: Glucocorticoids; RA: Rheumatoid arthritis; Exo: Exosome; FA: Folic acid; Dex: Dexamethasone sodium phosphate; PEG: Polyethylene glycol; Chol: Cholesterol; ELISA: Enzyme-linked immunosorbent assay; CIA: Collageninduced arthritis; EE: Encapsulation efficiency; DLE: Drug loading efficiency; ELVIS: Extravasation through leaky vasculature and subsequent inflammatory cell-mediated sequestration; Lip: Liposome; PDI: Polydispersity index; TEM: Transmission electron microscopy; HUVEC: Human umbilical vein endothelial cells; MTT: 3-(4,5dimethylthiozol-2-yl)-2,5-diphenyl-tetrazolium bromide; LPS: Lipopolysaccharide; Micro-CT: Micro-computed tomography; DID: 1,1'-dioctadecyl-3,3,3',3'-etramethylindodicarbocyanine perchlorate; Al: Articular index; H\&E: Hematoxylin-eosin staining; SO: Safranin O staining; AST: Aspartate aminotransferase; ALT: Alanine transaminase.

\section{Acknowledgements}

All authors are thankful for getting help and supports from the following research platforms, the Key Laboratory of Medical Electrophysiology of Ministry of Education, the Experimental Center of Basic Medicine, School of Pharmacy, Southwest Medical University, Luzhou, Sichuan 646000, China.

\section{Authors' contributions}

FY and YW conducted most of the research and ZZ wrote the manuscript, $Y F, Z M, H L$ and $X C$ helped with the experiment. $L C$ and $C L$ guided the entire study and edited the manuscript. All authors read and approved the final manuscript.

\section{Funding}

This work was supported by the National Natural Science Foundation of China (grant numbers 81803478, 2018); Science and Technology Project of Luzhou Government (grant numbers 2019-JYJ-51, 2019); Science and Technology Project of the Health Planning Committee of Sichuan (grant numbers 18ZD036, 2018); Sichuan Science and Technology Program (grant numbers 2017RZ0048, 2017); and the Transformation Project of Science and Technology Achievements of Southwest Medical University (grant numbers 2018002, 2018).

\section{Availability of data and materials}

All data generated or analyzed during this study are included in this article.

\section{Ethics approval and consent to participate}

Not applicable for this study.

\section{Consent for publication}

We give our consent for the manuscript to be published in Journal of Nanobiotechnology.

\section{Competing interests}

The authors declare that they have no competing interests.

\section{Author details}

${ }^{1}$ Department of Pharmaceutical Sciences, School of Pharmacy, Southwest Medical University, 3-319 Zhongshan Road, 646000 Luzhou, Sichuan, People's Republic of China. ${ }^{2}$ Department of Nuclear Medicine, The Affiliated Hospital of Southwest Medical University, 3-319 Zhongshan Road, 646000 Luzhou, Sichuan, People's Republic of China. ${ }^{3}$ Nuclear Medicine and Molecular Imaging Key Laboratory of Sichuan Province, 646000 Luzhou, Sichuan, China. ${ }^{4}$ The Research Center for Preclinical Medicine, Southwest Medical University, 646000 Luzhou, Sichuan, China. ${ }^{5}$ Engineering Research Center in Biomaterials, Sichuan University, 610064 Chengdu, Sichuan, People's Republic of China.

Received: 3 June 2020 Accepted: 11 August 2020

Published online: 20 August 2020

\section{References}

1. Prasad LK, O'Mary H, Cui Z. Nanomedicine delivers promising treatments for rheumatoid arthritis. Nanomed. 2015;10:1-12.

2. Quan L, Zhang Y, Crielaard BJ, Dusad A, Lele SM, Rijcken CJF, Metselaar JM, Kostkova H, Etrych T, Ulbrich K, et al. Nanomedicines for inflammatory arthritis: head-to-head comparison of glucocorticoid-containing polymers, micelles, and liposomes. ACS Nano. 2014;8:458-66.

3. Wang $Q$, Sun X. Recent advances in nanomedicines for the treatment of rheumatoid arthritis. Biomater Sci. 2017;5:1407-20.

4. Leblond A, Allanore Y, Avouac J. Targeting synovial neoangiogenesis in rheumatoid arthritis. Autoimmun Rew. 2017;16:594-601.

5. Wang Q, Jiang H, Li Y, Chen W, Li H, Peng K, Zhang Z, Sun X. Targeting NF-kB signaling with polymeric hybrid micelles that co-deliver siRNA and dexamethasone for arthritis therapy. Biomaterials. 2017;122:10-22.

6. Barnes PJ. Anti-inflammatory Actions of Glucocorticoids: Molecular Mechanisms. Clin Sci. 1998;94:557-72.

7. Vandewalle J, Luypaert A, Bosscher KD, Libert C. Therapeutic mechanisms of glucocorticoids. Trends Endocrinol Metab. 2017;29:42-544.

8. Wang Q, Jiang J, Chen W, Jiang H, Zhang Z, Sun X. Targeted delivery of low-dose dexamethasone using PCL-PEG micelles for effective treatment of rheumatoid arthritis. JCR. 2016;230:64-72.

9. Wang Y, Liu Z, Li T, Chen L, Lyu J, Li C, Lin Y, Hao N, Zhou M, Zhong Z. Enhanced therapeutic effect of RGD-modified polymeric micelles loaded with low-dose methotrexate and nimesulide on rheumatoid arthritis. Theranostics. 2019;9:708-20.

10. Li C, Li H, Wang Q, Zhou M, Li M, Gong T, Zhang Z, Sun X. pH-sensitive polymeric micelles for targeted delivery to inflamed joints. JCR. 2017;246:133-41.

11. Zhou M, Hou J, Zhong Z, Hao N, Lin Y, Li C. Targeted delivery of hyaluronic acid-coated solid lipid nanoparticles for rheumatoid arthritis therapy. Drug Deliv. 2018;25:716-22.

12. Li C, Wang J, Wang Y, Gao H, Wei G, Huang Y, Yu H, Gan Y, Wang Y, Mei L, Chen H, Hu H, Zhang Z, Jin Y. Recent progress in drug delivery. Acta Pharm Sin B. 2019:9:1145-62.

13. Holland CK, Mcpherson DD. Echogenic Lipsomes for Targeted Drug Delivery. Proc IEEE Int Symp Biomed Imaging. 2009;29:755-8.

14. Torchilin VP. Affinity lipsomes in vivo: factors influencing target accumulation. J Mol Recognit. 1996;9:335-46.

15. Wu G, Zhang J, Zhao Q, Zhuang W, Ding J, Zhang C, Gao H, Pang D, Pu $\mathrm{K}$, Xie H. Molecularly engineered macrophage-derived exosomes with inflammation tropism and intrinsic heme biosynthesis for atherosclerosis treatment. Angew Chem Int Ed Engl. 2020;59:4068-74.

16. Koenders MI, VandenBerg WB. Novel therapeutic targets in rheumatoid arthritis. Trends Pharmacol Sci. 2015;36:189-95.

17. Bunggulawa EJ, Wang W, Yin T, Wang N, Durkan C, Wang Y, Wang G. Recent advancements in the use of exosomes as drug delivery systems. J Nanobiotechnol. 2018;16:81-94.

18. Qu M, Lin Q, Huang L, Fu Y, Wang L, He S, Fu Y, Yang S, Zhang Z, Zhang $L$, Sun $X$. Dopamine-loaded blood exosomes targeted to brain for better treatment of Parkinson's disease. JCR. 2018;287:156-66.

19. Haney MJ, Klyachko NL, Zhao Y, Gupta R, Plotnikova EG, He Z, Patel T, Piroyan A, Sokolsky M, Kabanov AV, Batrakova EV. Exosomes as drug delivery vehicles for Parkinson's disease therapy. JCR. 2015;207:18-30. 
20. Yuan D, Zhao Y, Banks WA, Bullock KM, Haney M, Batrakova E, Kabanov AV. Macrophage exosomes as natural nanocarriers for protein delivery to inflamed brain. Biomaterials. 2017;142:1-12.

21. Lin Q, Qu M, Zhou B, Patra HK, Sun Z, Luo Q, Yang W, Wu Y, Zhang Y, $\mathrm{Li} L$, et al. Exosome-like nanoplatform modified with targeting ligand improves anti-cancer and anti-inflammation effects of imperialine. JCR. 2019;311:104-16.

22. Tang TT, Lv LL, Wang B, Cao JY, Feng Y, Li ZL, Li ZL, Wu M, Wang FM, Wen $Y$, et al. Employing macrophage-derived microvesicle for kidneytargeted delivery of dexamethasone: an efficient therapeutic strategy against renal inflammation and fibrosis. Theranostics. 2019;9:4740-55.

23. Malhi SS, Budhiraja A, Arora S, Chaudhari KR, Nepali K, Kumar R, Sohi $\mathrm{H}$, Murthy RSR. Intracellular delivery of redox cycler-doxorubicin to the mitochondria of cancer cell by folate receptor targeted mitocancerotropic liposomes. Int J Pharm. 2012;432:63-74.

24. Thomas TP, Goonewardena SN, Majoros IJ, Kotlyar A, Cao Z, Leroueil PR, Baker JR. Folate-targeted nanoparticles show efficacy in the treatment of inflammatory arthritis. Arthritis Rheum. 2011;63:2671-780.

25. Kinne RW, Bräuer R, Stuhlmüller B, Palombo-Kinne E, Burmester G-R. Macrophages in rheumatoid arthritis. Arthritis Res Ther. 2000:2:189.

26. Chiba M. Exosomes secreted from human colorectal cancer cell lines contain mRNAs, microRNAs and natural antisense RNAs, that can transfer into the human hepatoma HepG2 and lung cancer A549 cell lines. Oncol Rep. 2012:28:1551-8.

27. Siafaka P, Betsiou M, Tsolou A, Angelou E, Bikiari D. Synthesis of folatepegylated polyester nanoparticles encapsulating ixabepilone for targeting folate receptor overexpressing breast cancer cells. Deliv sys. 2015;26:275-89.

28. Saul JM, Annapragada A, Natarajan JV, Bellamkonda RV. Controlled targeting of liposomal doxorubicin via the folate receptor in vitro. JCR. 2003:92:49-67.

29. Yang KK, Kong M, Wei YN, Liu Y, Cheng XJ, Li J, Park HJ, Chen XG. Folatemodified-chitosan-coated liposomes for tumor-targeted drug delivery. J Mater Sci. 2013;48:1717-28.

30. Quan L, Zhang Y, Dusad A, Ren K, Purdue PE, Goldring SR, Wang D. The Evaluation of the Therapeutic Efficacy and Side Effects of a Macromolecular Dexamethasone Prodrug in the Collagen-Induced Arthritis Mouse Model. Pharm Res. 2016:33:186-93.

31. Wu J, Xin W, Gang Z, Josselyn G, Xiaoyan W, Liu Y, Dhruvkumar MS, Purdue PE, Mikuls TR, Goldring SR, Wang D. Development of a Janus Kinase (JAK) Inhibitor Prodrug for the Treatment of Rheumatoid Arthritis. Mol Pharm. 2018:15:456-3467.

32. Reumson A, Kim DY, Hun PS, Yong J, Kim K, Ju KB, Yin XY, Kim JH, Min $\mathrm{BH}, \mathrm{Han}$ DK, Kim MS. Direct chemotherapeutic dual drug delivery through intra-articular injection for synergistic enhancement of rheumatoid arthritis treatment. Sci Rep. 2015;5:147-53.

33. Crielaard BJ, Rijcken CJF, Quan L, Vander WS, Altintas I, Pot MVD, Kruijtzer JAW, Liskamp RMJ, Schiffelers RM, Nostrum CFV, et al. Glucocorticoid-loaded core-cross-linked polymeric micelles with tailorable release kinetics for targeted therapy of rheumatoid arthritis. Angew Chem Int Ed Engl. 2012;51:7254-8.

34. Zhao L, Gu C, Gan Y, Shao L, Chen H, Zhu H. Exosome-mediated siRNA delivery to suppress postoperative breast cancer metastasis. JCR. 2020;318:1-15

35. Beez CM, Haag M, Klein O, Sophie VL, Michael S, Martina S. Extracellular vesicles from regenerative human cardiac cells act as potent immune modulators by priming monocytes. J Nanobiotechnol. 2019;17:72-90.

36. Singh PP, Smith VL, Karakousis PC, Schorey JS. Exosomes Isolated from Mycobacteria-Infected Mice or Cultured Macrophages Can Recruit and Activate Immune Cells In Vitro and In Vivo. J Immunol. 2012;189:777-85.
37. Zhang Z, Wang C, Li T, Liu Z, Li L. Comparison of ultracentrifugation and density gradient separation methods for isolating Tca8113 human tongue cancer cell line derived exosomes. Oncol Lett. 2014;8:1701-6.

38. Yamashita T, Takahashi Y, Nishikawa M, Takakura Y. Effect of Exosome isolation methods on physicochemical properties of exosomes and clearance of exosomes from the blood circulation. Euro J of Pharm Biopharm. 2015:98:1-8.

39. Lai RC, Yeo RWY, Tan KH, Lim SK. Exosomes for drug delivery-a novel application for the mesenchymal stem cell. Biotechnol Adv. 2012;31:543-51.

40. Johnsen KB, Gudbergsson JM, Skov MN, Christiansen G, Gurevich L, Moos T, Duroux M. Evaluation of electroporation-induced adverse effects on adipose-derived stem cell exosomes. Cytotechnology. 2016;68:2125-38.

41. Hood JL, Scott MJ, Wickline SA. Maximizing exosome colloidal stability following electroporation. Anal Biochem. 2014;448:41-9.

42. Kooijmans SAA, Fliervoet LAL, van der Meel R, Fens MHAM, Heijnen HFG, van Bergen, Henegouwen PMP, Vader P, Schiffelers R M. PEGylated and targeted extracellular vesicles display enhanced cell specificity and circulation time. JCR. 2016; 224: 77-85.

43. Ju J. Temporal differential effects of proinflammatory cytokines on osteoclastogenesis. Int J Mol Med. 2013;31:769-77.

44. Pathak JL, Bravenboer N, Verschueren P, Lems WF, Luyten FP, Klein-Nulend J, Bakker AD. Inflammatory factors in the circulation of patients with active rheumatoid arthritis stimulate osteoclastogenesis via endogenous cytokine production by osteoblasts. Osteoporos Int. 2014;25:2453-63.

45. Son HS, Lee J, Lee HI, Kim N, Jo YJ, Lee GR, et al. Benzydamine inhibits osteoclast differentiation and bone resorption via down-regulation of interleukin-1 $\beta$ expression. Acta Pharm Sin B. 2020;10:462-74.

46. Yang T, Martin P, Fogarty B, Brown A, Schurman K, Phipps R. Exosome delivered anticancer drugs across the blood-brain barrier for brain cancer therapy in Danio Rerio. Pharmac Res. 2015;32:2003-144.

47. Tian Y, Li S, Song J, Ji T, Zhu M, Anderson GJ. A doxorubicin delivery platform using engineered natural membrane vesicle exosomes for targeted tumor therapy. Biomaterials. 2014;35:2383-90.

48. Kim MS, Haney MJ, Zhao Y, Mahajan V, Batrakova EV. Development of Exosome-encapsulated Paclitaxel to Overcome MDR in Cancer cells. Nanomed. 2015;12:655-64.

49. Yan F, Li H, Zhong Z, Zhou M, Lin Y, Tang C, Li C. Co-Delivery of Prednisolone and Curcumin in Human Serum Albumin Nanoparticles for Effective Treatment of Rheumatoid Arthritis. Int J Nanomedicine. 2019;14:9113-255.

50. Liu C, Zhang W, Li Y, Chang J, Sun J. Microfluidic sonication to assemble exosome membrane-coated nanoparticles for immune evasion-mediated targeting. Nano Lett. 2019;19:7836-44.

51. Petro Z, Alexander E, Thomas B, Anastasia M, Phil R, Claudia M, Anja R, Yarua J, Achim A. Extracellular vesicle (ECV)-modified polyethylenimine (PEI) complexes for enhanced siRNA delivery in vitro and in vivo. JCR. 2020;319:63-766

\section{Publisher's Note}

Springer Nature remains neutral with regard to jurisdictional claims in published maps and institutional affiliations.

Ready to submit your research? Choose BMC and benefit from

- fast, convenient online submission

- thorough peer review by experienced researchers in your field

- rapid publication on acceptance

- support for research data, including large and complex data types

- gold Open Access which fosters wider collaboration and increased citations

- maximum visibility for your research: over 100M website views per year

At BMC, research is always in progress.

Learn more biomedcentral.com/submissions 